\title{
Dynamics of a Ferromagnetic Domain Wall and the Barkhausen Effect
}

\author{
Pierre Cizeau, ${ }^{1}$ Stefano Zapperi, ${ }^{1}$ Gianfranco Durin,${ }^{2}$ and H. Eugene Stanley ${ }^{1}$ \\ ${ }^{1}$ Center for Polymer Studies and Department of Physics, Boston University, Boston, Massachusetts 02215 \\ ${ }^{2}$ Istituto Elettrotecnico Nazionale Galileo Ferraris and GNSM-INFM, Corso M. d'Azeglio 42, I-10125 Torino, Italy
}

(Received 2 July 1997)

\begin{abstract}
We derive an equation of motion for the dynamics of a ferromagnetic domain wall driven by an external magnetic field through a disordered medium, and we study the associated depinning transition. The long-range dipolar interactions set the upper critical dimension to be $d_{c}=3$, so we suggest that mean-field exponents describe the Barkhausen effect for three-dimensional soft ferromagnetic materials. We analyze the scaling of the Barkhausen jumps as a function of the field driving rate and the intensity of the demagnetizing field, and find results in quantitative agreement with experiments on crystalline and amorphous soft ferromagnetic alloys. [S0031-9007(97)04766-2]
\end{abstract}

PACS numbers: 75.60.Ej, 68.35.Ct, 75.60.Ch

The magnetization of a ferromagnet displays discrete jumps as the external magnetic field is increased. This phenomenon, known as the Barkhausen effect, was first observed in 1919 by recording the tickling noise produced by the sudden reversal of the Weiss domains [1]. The Barkhausen effect has been widely used as a nondestructive method to test magnetic materials, and the statistical properties of the noise have been analyzed in detail $[2,3]$. In particular, it has been observed that the distributions of sizes and durations of Barkhausen jumps decay as power laws at low applied field rates [4-6]. In addition to its practical and technological applications, the Barkhausen effect has recently attracted a growing interest as an example of a complex dynamical system displaying critical behavior [7-11].

In soft ferromagnetic materials the magnetization process is composed of two distinct mechanisms [12]. (i) When the field is increased from the saturated region, domains nucleate in the sample, typically starting from the boundaries. (ii) In the central part of the hysteresis loop, around the coercive field, the magnetization process is due mainly to domain wall motion. The disorder present in the material (due to nonmagnetic impurities, lattice dislocations, residual stresses, etc.) is responsible for the jerky motion of the domain walls, giving rise to the jumps observed in the magnetization. The moving walls are usually parallel to the magnetization $\left(180^{\circ}\right.$ walls), and span the sample from end to end [12]. The statistical properties of the Barkhausen effect are normally studied in the central part of the hysteresis loop and can therefore be understood by studying domain wall motion.

It has recently been proposed to relate the scaling properties of the Barkhausen noise to the critical behavior expected at the depinning transition of an elastic interface [9,11]. The numerical values of the scaling exponents, however, do not agree with most experimental data [4-6]. Interestingly, a quantitative description of the phenomenon can be obtained by a simple phenomenological model, where the wall is described as a single point moving in a correlated random pinning field [13].
Here, we present an accurate treatment of magnetic interactions in the context of the depinning transition, which allows us to explain the experiments and to give a microscopic justification for the model of Ref. [13]. We study a single domain wall separating two regions with magnetization of constant magnitude $M$ and opposite directions. We assume that the wall surface does not form overhangs, and describe the position of the wall by its height $h(\vec{r}, t)$. The motion of the wall is overdamped because of eddy currents so, neglecting thermal fluctuations, the evolution of $h(\vec{r}, t)$ is governed by

$$
\frac{\partial h(\vec{r}, t)}{\partial t}=-\frac{\delta E(\{h(\vec{r}, t)\})}{\delta h(\vec{r}, t)},
$$

where $E(\{h(\vec{r}, t)\})$ is the energy of the system [14,15]. We will show that by incorporating the effects of ferromagnetic, magnetocrystalline, magnetostatic and dipolar interactions, and disorder, the equation of motion is

$$
\begin{aligned}
\frac{\partial h(\vec{r}, t)}{\partial t}= & \nu_{0} \nabla^{2} h(\vec{r}, t)+H-H_{d}+\int d^{2} r^{\prime} K\left(\vec{r}-\vec{r}^{\prime}\right) \\
& \times\left[h\left(\vec{r}^{\prime}, t\right)-h(\vec{r}, t)\right]+\eta(\vec{r}, h) .
\end{aligned}
$$

Here $\nu_{0}$ is the surface tension, $H$ is the external magnetic field, the demagnetizing field $H_{d}$ and the nonlocal term are due to dipolar interactions, and we model the disorder with a random force, with Gaussian distribution and short range correlations,

$$
\left\langle\eta(\vec{r}, h) \eta\left(\vec{r}^{\prime}, h^{\prime}\right)\right\rangle=\delta^{2}\left(\vec{r}-\vec{r}^{\prime}\right) \Delta\left(h-h^{\prime}\right),
$$

where $\Delta(x)$ decays very rapidly for large values of the argument.

The dipolar interactions are treated considering effective magnetic charges induced by the discontinuities of the magnetization across the boundaries of the sample and the domain wall [16]. The corresponding magnetic surface charge is given by

$$
\sigma=\left(\vec{M}_{1}-\vec{M}_{2}\right) \cdot \hat{n}
$$


where $\hat{n}$ is normal to the surface and $\vec{M}_{1}$ and $\vec{M}_{2}$ are the magnetization vectors on each side of the surface. The surface charge induced at the boundary of the sample gives rise to a demagnetizing field which opposes the external field [12]. The simplest approximation is to consider this field to be constant throughout the sample, and to be proportional to the total magnetization $[9,13]$. This yields the term $H_{d}=-k \int d^{2} r h(\vec{r}, t)$ in Eq. (2), where the demagnetizing factor $k$ takes into account the geometry of the domain structure, the shape of the system, and its size.

Magnetic surface charges will also appear on the domain wall when its surface is not parallel to the magnetization. In the limit of infinite anisotropy [17] and small bending of the surface, we can express the surface charge as

$$
\sigma(\vec{r})=2 M \cos \theta \simeq 2 M \frac{\partial h(\vec{r}, t)}{\partial x},
$$

where $\theta$ is the local angle between the vector normal to the surface and the magnetization (see Fig. 1). The interaction energy of these charges $E_{d}=(1 / 2) \int d^{2} r d^{2} r^{\prime} \times$ $\sigma(\vec{r}) \sigma\left(\vec{r}^{\prime}\right) /\left|\vec{r}-\vec{r}^{\prime}\right|$ gives rise to the nonlocal kernel present in Eq. (2) $[14,15,18]$ :

$$
K\left(\vec{r}-\vec{r}^{\prime}\right)=\frac{2 M^{2}}{\left|\vec{r}-\vec{r}^{\prime}\right|^{3}}\left(1+\frac{3\left(x-x^{\prime}\right)^{2}}{\left|\vec{r}-\vec{r}^{\prime}\right|^{2}}\right) .
$$

The interaction (6) is long range and anisotropic, as can also be seen by considering the Fourier transform

$$
K(p, q)=\frac{2 M^{2}}{\pi} \frac{p^{2}}{\sqrt{p^{2}+q^{2}}},
$$

where $p$ and $q$ are the two components of the Fourier vector. Moreover, an estimate of the order of magnitude of $K\left(\vec{r}-\vec{r}^{\prime}\right)$ shows that it dominates over the surface tension for all length scales of interest [19].

Apart from the nonlocal kernel, Eq. (2) is the equation proposed in Ref. [9] which, in turn, reduces when $k=0$ to a driven elastic interface in the presence of quenched disorder [20-22].

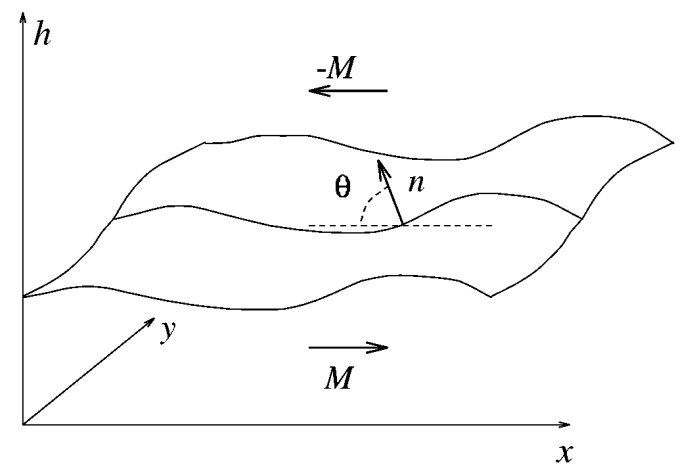

FIG. 1. The domain wall separating two regions of opposite magnetization. The discontinuities of the normal component of the magnetization across the domain wall generate magnetic charges.
For $k=0$ (no demagnetizing field), Eq. (2) displays a depinning transition; i.e., there exists a critical field $H_{c}$ such that for $H<H_{c}$ the interface is pinned while for $H>H_{c}$, it moves with nonzero average velocity. At $H=H_{c}$, the system exhibits scaling properties: The interface moves by avalanches whose sizes $s$ and durations $T$ distributions follow power laws,

$$
P(s) \sim s^{-\tau}, \quad P(T) \sim T^{-\alpha} .
$$

When $k>0$, the demagnetizing field provides an additional restoring force that keeps the interface at the depinning transition $[9,11]$ if the external field is increased adiabatically.

Using the functional renormalization group scheme introduced in Ref. [23], we find that-due to the longrange kernel $K\left(\vec{r}-\vec{r}^{\prime}\right)$ in Eq. (6) - the critical behavior of Eq. (2) differs from that of elastic interfaces: The upper critical dimension becomes $d_{c}=3[18,24,25]$, instead of $d_{c}=5[21,22]$. Hence we predict that, for $d=3$, the motion of the domain wall will be described by mean-field theory (apart from logarithmic corrections), which yields $[18,22] \tau=3 / 2, \alpha=2$, and that the surface is flat (the roughness exponent $\zeta$ is zero). These values differ significantly from the results of elastic interfaces which in $d=3$ are $\tau \simeq 1.3, \alpha \simeq 1.5$, and $\zeta \simeq 0.7[22,26]$.

Next we make contact between our approach and the conventional approach that reduces the domain wall to a single point moving in a random pinning field $[3,13,27]$. To this end, we introduce an infinite range version $(d \rightarrow \infty)$ of Eq. (2), which should have the same critical behavior as Eq. (2) but has the advantage of being much simpler to analyze.

To treat the infinite-range model, we discretize the interface and consider that all $N$ elements are at the same distance from each other. Equation (2) then becomes [28]

$$
\frac{\partial h_{i}(t)}{\partial t}=H(t)-\chi \bar{h}+J\left[\bar{h}-h_{i}(t)\right]+\eta_{i}(h),
$$

where $\bar{h} \equiv \sum_{i=1}^{N} h_{i} / N, \chi \equiv N k, J \equiv\left(\nu_{0}+2 M^{2}\right)$, and the external field $H(t)$ increases at a finite constant rate. Summing Eq. (9) over all sites $i$, we obtain an equation for the total magnetization $m \equiv N \bar{h}$,

$$
\frac{d m}{d t}=\tilde{c} t-\chi m+\sum_{i=1}^{N} \eta_{i}(h)
$$

where the time dependence of the field has been made explicit. We can approximate $\sum \eta_{i}$ by an effective random pinning field $W(m)$, depending only on the magnetization. When the interface moves between two configurations, the change in $W$ is

$$
W\left(m^{\prime}\right)-W(m)=\sum_{i}^{\prime} \Delta \eta_{i},
$$

where the sum is restricted to the sites that have moved (i.e., their disorder is changed). The total number of such 
sites scales, on average, as $\left|m^{\prime}-m\right|$, since for $d \geq d_{c}$ the size of an avalanche scales like its area [18,22]. Assuming that the $\Delta \eta_{i}$ are uncorrelated and have random signs, we find that the effective pinning field is correlated,

$$
\left\langle\left|W\left(m^{\prime}\right)-W(m)\right|^{2}\right\rangle=D\left|m^{\prime}-m\right|,
$$

where $D$ sets the scale of the fluctuations of $W$. These correlations of "Brownian" type have been experimentally observed in $\mathrm{SiFe}$ alloys [3]. In the model of Alessandro et al. [13] (ABBM), the domain wall is treated as a single point moving in a Brownian correlated field. The ABBM model is equivalent to Eqs. (10) and (12), and predicts that the avalanche exponents should depend on the field driving rate, with $\tau=3 / 2-c / 2$ and $\alpha=2-c$ [5], where $c \equiv$ $\tilde{c} / D$. In the adiabatic limit $c \rightarrow 0$, we recover the meanfield exponents of the depinning transition. Moreover, our results imply that Brownian correlations in the pinning field do not reflect peculiar long-range correlations in the impurities, but represent an effective description of the collective motion of the interface.

To confirm the results obtained above, we first simulate an automaton version of Eq. (2) in three dimensions, applying an adiabatically increasing magnetic field. In this limit, we recover, for the distribution of avalanche sizes and durations, the results expected for the depinning transition in mean-field theory [18].

To overcome the numerical limitations posed by Eq. (2) and obtain an extensive avalanche statistics as a function of $c$ and $\chi$, we next simulate the infinite-range model of Eq. (9). We measure the distributions of domain wall velocities and avalanche sizes and durations (see Figs. 2 and 3 , and [18]). For $c<1$, we find power laws with exponents in perfect agreement with the ABBM model. The cutoff of the distributions is determined by the demagnetizing fields and diverges for $\chi \rightarrow 0$ [18]. In the case of the avalanche size distribution, it scales as $\chi^{-2}$ (see Fig. 3).

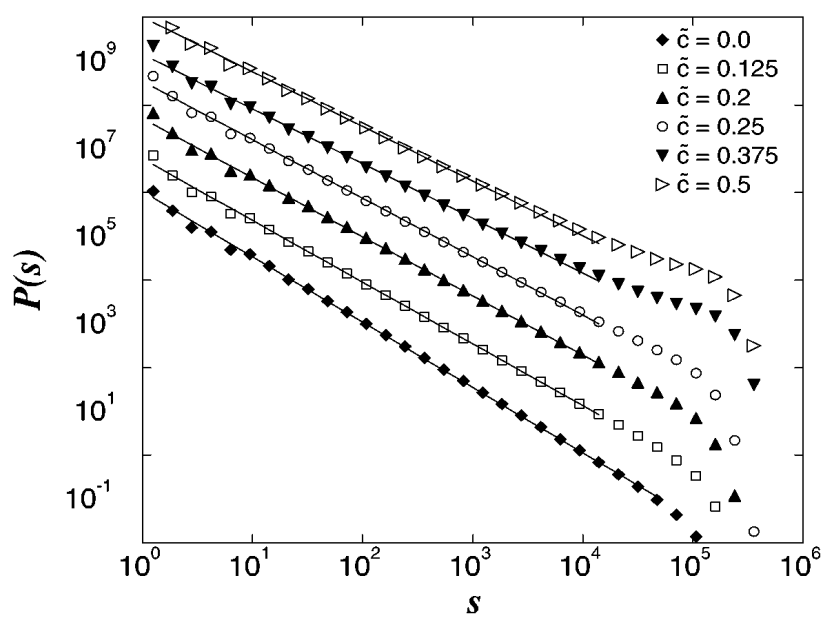

FIG. 2. The distribution of avalanche sizes in the infiniterange model as a function of $c$ for $N=32696, \chi=0.0075$. The lines are the theoretical predictions $\tau=3 / 2-c / 2$.
It would be interesting to experimentally study the behavior of the cutoff by externally changing the demagnetizing field.

Our results are in agreement with experiments on crystalline $(\mathrm{SiFe})[4,5]$ and amorphous (Co-base and $\mathrm{Fe}$ base) ferromagnetic alloys [29] performed at different values of $c$, which yield $\tau=3 / 2-c / 2$ and $\alpha=2-$ $c$. A direct comparison with experiments in which the parameter $c$ is not defined, as in the case of a sinusoidal driving field, is problematic [6,7]. The result presented in Ref. [9] (i.e., $\tau \simeq 1.3$ ) could be due to a finite driving rate $(c \simeq 0.4)$. In addition, it is important to remark that the present theory applies only if domain wall motion is the dominant magnetization process. This was indeed the case in Refs. [4,5,29], where the noise was recorded only in the region of constant permeability around the coercive field. A detailed critical discussion of the experimental results reported in the literature can be found in Ref. [29].

In particular geometries, typically frames or toroid samples, the demagnetizing field is absent $(\chi=0)$ [3]. It is then possible to observe experimentally the depinning transition of domain walls. It has been reported that the average velocity of the domain walls, in different ferromagnetic materials [30], increases for $H>H_{c}$, as

$$
v \sim\left(H-H_{c}\right)^{\beta},
$$

with $\beta=1$, in agreement with the theory $(\beta=1$ is expected in mean-field theory [22]).

We have seen that avalanche distributions can be described by power laws with exponents that do not depend on material details. The power spectrum of the noise displays instead a more complex structure and does not show such a marked universality. At low frequency the power spectrum grows with an exponent varying between $\psi=0.5$ for crystalline alloys and $\psi=1$ for amorphous

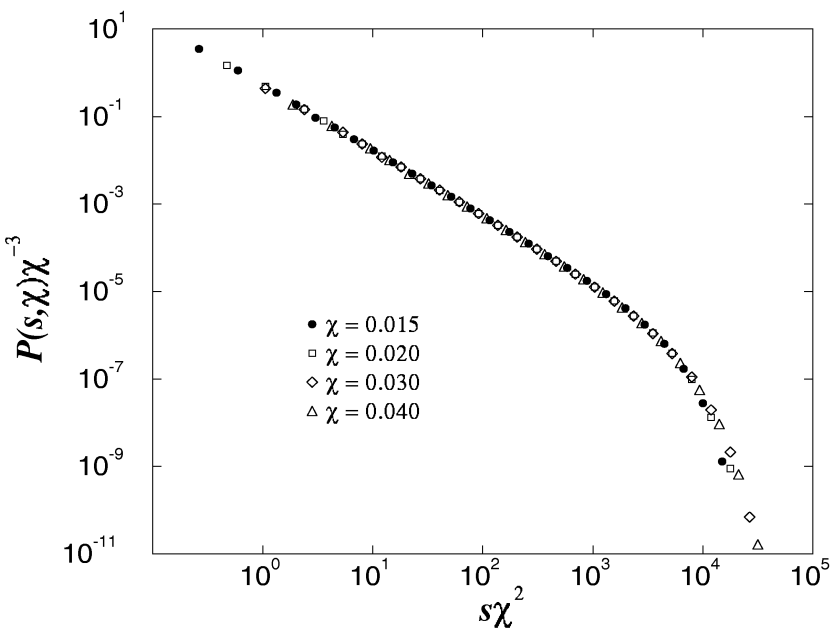

FIG. 3. The distribution of avalanche sizes in the infinite range model as a function of $\chi$ for $N=32696$ and $\tilde{c}=$ 0 . The data collapse is obtained from the scaling function $P(s, \chi)=s^{-3 / 2} f\left(\chi^{2} s\right)$. 
alloys, while at high frequencies it decays with an exponent varying between $\psi=-2$ for crystals and $\psi=-1.6$ for amorphous alloys [2,4-6,29]. In samples with a single domain wall present, the power spectrum was found to decay as $\omega^{-2}[3]$.

Following the analysis of Tang et al. [31], we obtain, at low frequency, $\psi=1$ for the power spectrum measured on a single site and $\psi=0$ when the signal is averaged over the whole system. For the averaged spectrum, we also find a $\omega^{-2}$ decay at large frequencies, due to the Brownian properties of the effective pinning field. The discrepancies between theory and experiments could be due to the presence of many domain walls interacting through the demagnetizing field. When a domain wall starts to move, the demagnetizing field increases, creating a larger pinning force on the other walls. Therefore, on short time scales the interactions between the walls is irrelevant and should not change the avalanche distributions. On larger time scales, this effect may be important and could modify the properties of the power spectrum. In order to clarify this issue, it would be necessary to analyze in detail the dynamics of many coupled domain walls.

The present theory for the Barkhausen effect, based on the depinning of a ferromagnetic domain wall, should apply to soft ferromagnetic materials, which are frequently used in experimental studies of the Barkhausen effect [2$6,9,13]$. For hard ferromagnets and rare earth materials where strong random anisotropies prevent the formation of domains, disordered spin models could be appropriate [8].

We thank G. Bertotti, J.-P. Bouchaud, D. Ertas, M. Mézard, S. Milošević, and S. Roux for useful discussions, and L. A. N. Amaral and M. Meyer for critical reading of the manuscript. The Center for Polymer Studies is supported by NSF.

[1] H. Barkhausen, Z. Phys. 20, 401 (1919).

[2] H. Bittel, IEEE Trans. Magn. 5, 359 (1969); J. A. Baldwin and G. M. Pickles, J. Appl. Phys. 43, 1263 (1972); W. Grosse-Nobis, J. Magn. Magn. Mater. 4, 247 (1977); K.P. O'Brien and M.B. Weissmann, Phys. Rev. E 50, 3446 (1994).

[3] R. Vergne, J. C. Cotillard, and J. L. Porteseil, Rev. Phys. Appl. 16, 449 (1981).

[4] G. Bertotti, G. Durin, and A. Magni, J. Appl. Phys. 75, 5490 (1994).

[5] G. Durin, G. Bertotti, and A. Magni, Fractals 3, 351 (1995).

[6] D. Spasojević, S. Bukvić, S. Milosević, and H. E. Stanley, Phys. Rev. E 54, 2531 (1996).

[7] P. J. Cote and L. V. Meisel, Phys. Rev. Lett. 67, 1334 (1991).
[8] J. P. Sethna, K. Dahmen, S. Karta, J. A. Krumhansl, and J. D. Shore, Phys. Rev. Lett. 70, 3347 (1993); O. Perkovic, K. Dahmen, and J.P. Sethna, ibid. 75, 4528 (1995).

[9] J. S. Urbach, R. C. Madison, and J. T. Markert, Phys. Rev. Lett. 75, 276 (1995).

[10] J. R. Petta, M. B. Weissman, and K. P. O'Brien, Phys. Rev. E 54, R1029 (1996).

[11] O. Narayan, Phys. Rev. Lett. 77, 3855 (1996).

[12] A. Herpin, Théorie du Magnetisme (Presse Universitaires de France, Paris, 1968).

[13] B. Alessandro, C. Beatrice, G. Bertotti, and A. Montorsi, J. Appl. Phys. 68, 2901 (1990); ibid. 68, 2908 (1990).

[14] L. Néel, Ann. Univ. Grenoble 22, 299 (1946).

[15] H. R. Hilzinger and H. Kronmüller, J. Magn. Magn. Mater. 2, 11 (1976).

[16] J.D. Jackson, Classical Electrodynamics (Wiley, New York, 1962).

[17] In the case of finite anisotropy, the direction of the magnetization can deviate from the easy axis. This effect can be included in the derivation, and the resulting kernel is qualitatively unchanged [14].

[18] S. Zapperi, P. Cizeau, G. Durin, and H. E. Stanley (to be published).

[19] The long-range kernel becomes relevant for length scales larger than $L \sim \nu_{0} / M^{2}$. In typical ferromagnets, $M \sim$ $10^{3}$ and $\nu_{0} \sim 1$ (in cgs units) (see page 713 of Ref. [12]). This implies $L \sim 10^{-6} \mathrm{~cm}$, which is of the order of the domain wall thickness.

[20] J. Koplik and H. Levine, Phys. Rev. B 32, 280 (1985).

[21] T. Nattermann, S. Stepanow, L. H. Tang, and H. Leschhorn, J. Phys. II (France) 2, 1483 (1992).

[22] O. Narayan and D. S. Fisher, Phys. Rev. B 48, 7030 (1993).

[23] O. Narayan and D. S. Fisher, Phys. Rev. Lett. 68, 3615 (1992); Phys. Rev. B 46, 11520 (1992).

[24] D. Ertas and M. Kardar, Phys. Rev. E 49, R2532 (1994); D. S. Fisher, K. Damen, S. Ramanathan, and Y. Ben-Zion, Phys. Rev. Lett. 78, 4885 (1997).

[25] Narayan [11] has considered dipolar interactions, concluding that they do not change the universality class of elastic interfaces, in contrast with our results.

[26] H. Leschhorn, T. Nattermann, S. Stepanow, and L. H. Tang, Ann. Phys. 6, 1 (1997).

[27] L. Néel, Cah. Phys. 12, 1 (1942); ibid. 13, 1 (1943).

[28] D. S. Fisher, Phys. Rev. B 31, 1396 (1985).

[29] G. Durin, Proceedings of the 14th International Conference on Noise in Physical Systems and 1/f Fluctuations (World Scientific, Singapore, 1997).

[30] H. J. Williams, W. Shockley, and C. Kittel, Phys. Rev. 80, 1090 (1950); J. K. Galt, Bell. Syst. Tech. J. 33, 1023 (1954); C. P. Bean and D. S. Rodbell, J. Appl. Phys. 26, 124 (1955); D. S. Rodbell and C. P. Bean, Phys. Rev. 103, 886 (1956).

[31] C. Tang, S. Feng, and L. Golubović, Phys. Rev. Lett. 72, 1264 (1994). 